\title{
Gal1 repression memory in budding yeast
}

\author{
Lea Schuh ${ }^{1,2,3,4}$, Igor Kukhtevich ${ }^{2}$, Poonam Bheda ${ }^{2}$, Melanie Schulz ${ }^{1,3}$, Maria Bordukova ${ }^{3}$, Robert \\ Schneider ${ }^{2,5,6, *}$, and Carsten Marr ${ }^{1,3,{ }^{*}}$ \\ ${ }^{1}$ Institute of Computational Biology, Helmholtz Zentrum München - German Research Center for Environmental \\ Health, 85764 Neuherberg, Germany \\ ${ }^{2}$ Institute of Functional Epigenetics, Helmholtz Zentrum München - German Research Center for Environmental \\ Health, 85764 Neuherberg, Germany \\ ${ }^{3}$ Institute of Al for Health, Helmholtz Zentrum München - German Research Center for Environmental Health, 85764 \\ Neuherberg, Germany \\ ${ }^{4}$ Department of Mathematics, Technical University of Munich, Garching, 85748, Germany \\ ${ }^{5}$ German Center for Diabetes Research (DZD), 85764 Neuherberg, Germany \\ ${ }^{6}$ Faculty of Biology, Ludwig-Maximilians University of Munich, 82152 Planegg-Martinsried, Germany \\ *correspondence: robert.schneider@helmholtz-muenchen.de, carsten.marr@helmholtz-muenchen.de
}

\begin{abstract}
Cells must continuously adapt to changing environments and, thus, have evolved mechanisms allowing them to respond to repeated stimuli. For example, faster gene induction upon a repeated stimulus aids adaptation - a process known as reinduction memory. However, whether such a memory exists for gene repression is unclear. Here, we studied gene repression across repeated carbon source shifts in over 2,500 single Saccharomyces cerevisiae cells. By monitoring the expression of a carbon source-responsive gene, galactokinase 1 (Gal1), and mathematical modeling, we discovered repression memory at the population and single-cell level. Using a repressor model to estimate single-cell repression parameters, we show that repression memory is due to a shortened repression delay, the estimated time gap between carbon source shift and Gal1 expression termination, upon the repeated carbon source shift. Additionally, we show that cells lacking Elp6 display a gain-of-repression-memory phenotype characterized by a stronger decrease in repression delay between two consecutive carbon source shifts. Collectively, our study provides the first quantitative description of repression memory in single cells.
\end{abstract}




\section{INTRODUCTION}

Cells receive and process external signals to optimally adapt to changing environments. Repeated stimulation from the same external signal induces an adapted transcriptional response, a phenomenon termed transcriptional memory (1). It is crucial to understand the mechanisms underlying transcriptional memory due to its implications for a broad range of cellular functions, including the human adaptive immune system $(2,3)$, disease development in diabetes $(4,5)$, and aging (6). However, transcriptional memory has primarily been researched concerning gene induction despite gene repression playing an essential role in gene regulation $(7,8)$. This raises the question of whether memory exists in repression.

The adaptation of Saccharomyces cerevisiae (budding yeast) to carbon sources is among the most well-studied eukaryotic signal integration systems. Whereas glucose directly enters glycolysis, a vital metabolic route providing cells with energy, galactose is first converted to glucose-6-phosphate $(9,10)$, necessitating the production of Gal gene-encoded enzymes (11). Repeated alternations between glucose and galactose media revealed that yeast cells are primed by their carbon source history, exhibiting transcriptional memory: repeated galactose induction results in enhanced Gal gene expression (12-16). Bheda et al. examined the expression of galactokinase 1 (Gal1) in single cells, for which reinduction memory has been well characterized, and discovered that a shorter delay, rather than an increased expression rate, contributed to the observed increase in Gal1 levels (17). Moreover, they identified elp6 $\Delta$ as a gain-of-reinduction memory mutant, with elp6 $\Delta$ cells showing Gal1 levels comparable to wildtype cells in the first induction, but earlier induction onset and increased Gal1 levels in the second induction. While, as these examples show, reinduction memory upon galactose induction has been thoroughly researched, and, while, Lee et al. suggested repression memory for bulk populations (18), it is uncertain if individual cells display Gal1 repression memory upon repeated glucose repression (Figure 1).

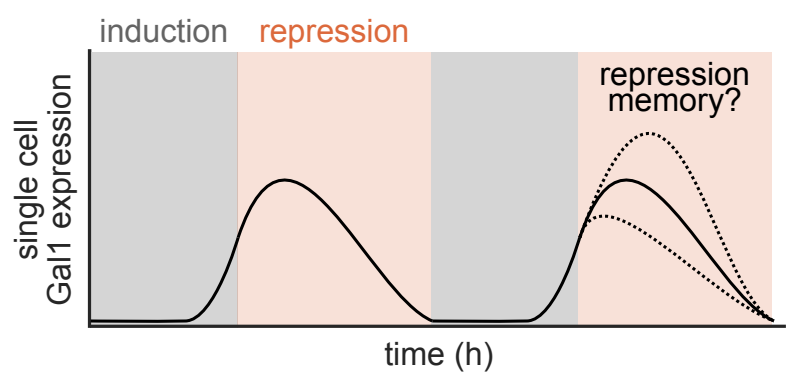

Figure 1. Does gene repression memory exist in budding yeast?

Previous studies focused on gene expression during induction. However, whether there is memory in repression indicated by altered repression kinetics is still unknown. 
We measured Gal1 expression in wildtype budding yeast cells via a Gal1-GFP (green fluorescent protein) fusion across repeated galactose inductions and glucose repressions using time-lapse microscopy coupled with a microfluidic device to follow and study Gal1 repression kinetics. In the second repression, we discovered that the time of maximal mean Gal1 expression was shortened. By using a mathematical model to quantify single-cell Gal1-GFP kinetics during glucose repression, and by distinguishing between repressor and non-repressor cells, we revealed that the shortened time to maximal mean Gal1 expression at the population level was not caused by different fractions of repressor cells between consecutive repressions. Using the estimated singlecell parameters, we found the repression delay, which is the estimated time gap between the galactose to glucose shift and the Gal1 expression termination, to be shortened in the second repression at the single-cell level, implying Gal1 repression memory. Furthermore, we repeated the experiments and analysis for the gain-of-induction memory mutant elp6 $\Delta$. Remarkably, elp6 $\Delta$ cells showed a stronger repression memory effect than wildtype, making elp6 $\Delta$ also a gain-ofrepression memory mutant.

\section{RESULTS}

\section{Automated time-lapse microscopy and microfluidics allow for the quantification of single- cell Gal1 repression kinetics across repeated carbon source shifts}

To study Gal1 repression kinetics over multiple repressions, we exposed wildtype budding yeast cells alternatingly to glucose or galactose media (Figure 2A). For this, we cultured the cells in custom-made microfluidic devices to ensure precise media shifts and long-term tracking (17). Gal1 expression levels in single yeast cells were monitored using a Gal1-GFP fusion, a standard reporter to study gene expression in time-lapse microscopy (see Bheda et al., 2020 for details). We captured images from the microfluidics chambers every 3 min totaling 320 images per chamber during a 16-h experiment. The yeast cells were then semi-automatically segmented, mapped and the total Gal1-GFP fluorescence signal per cell and time point was extracted using Autotrack and PhyloCell (19), YeaZ (20) and Cell-ACDC (21) (see Materials and Methods), yielding over 2,500 single-cell Gal1 expression traces (Figure 2B). Asymmetric budding allowed us to identify mother-daughter relationships. As expected, the total Gal1-GFP fluorescence signal of single cells reveals Gal1 inductions and repressions during galactose and glucose, respectively, and increased overall Gal1 levels in induction i2 (Figure 2B).

During budding, cytoplasmic proteins are disseminated between the mother and daughter cells. Assuming a constant Gal1 protein amount, its redistribution decreases the total Gal1-GFP fluorescence signal in the mother cell (Figure 2C top), a phenomenon called dilution. To deconvolute dilution and repression kinetics, we calculated the sum of the total Gal1-GFP fluorescence signal of the mother cell and its progeny (Figure $2 \mathrm{C}$ bottom, and see Materials and Methods). In the following, the adjusted sum of the total Gal1-GFP fluorescence signal of the mother cell and its progeny is referred to as total GFP. We applied the same dilution compensation to repressions $\mathrm{r} 1$ and $\mathrm{r} 2$ (Figure 2D). 
A

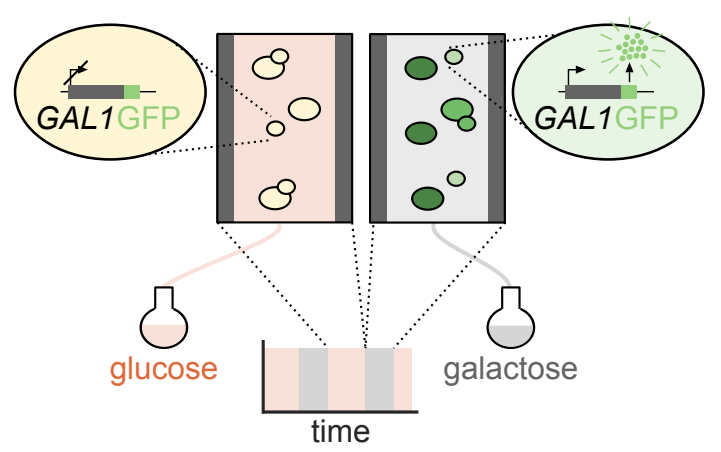

B

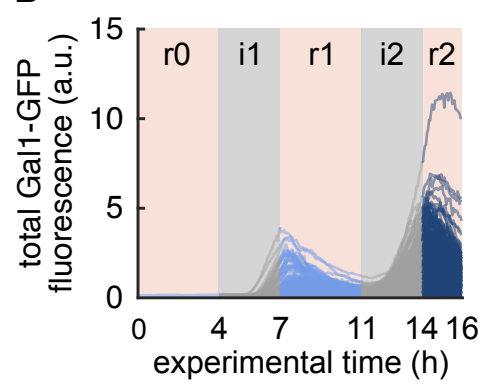

D

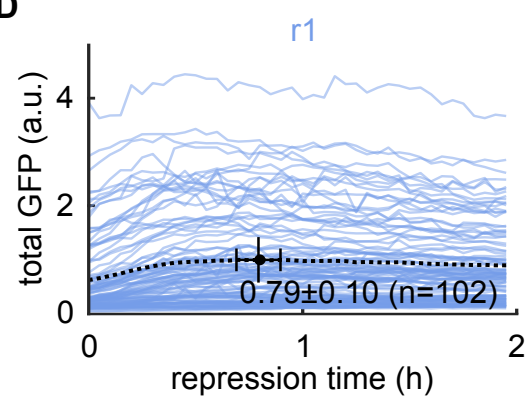

C

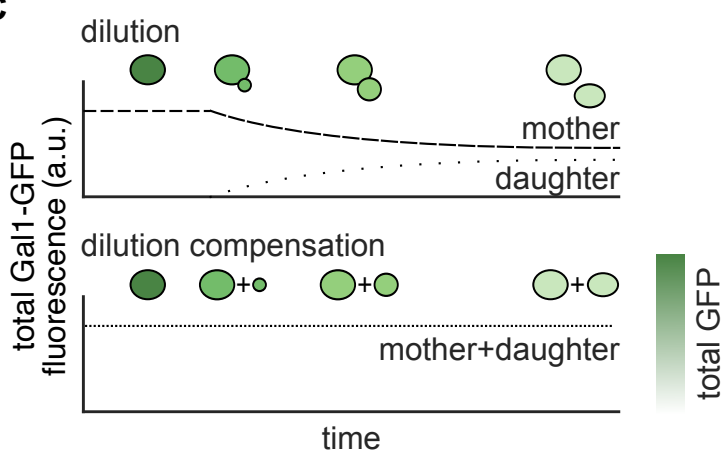

Figure 2. A shortened time to maximal mean total GFP in the second repression at the population level.

(A) Budding yeast cells were grown in microfluidic chambers and alternatingly exposed to a medium containing either glucose (orange) or galactose (gray) as carbon source. Galactokinase 1 (Ga/1) is induced in cells exposed to galactose and repressed in cells exposed to glucose. Gal1 expression was monitored via a Gal1-GFP fusion and time-lapse microscopy.

(B) Single-cell traces of total Gal1-GFP fluorescence signal across two inductions i1 and i2 (gray) and repressions $\mathrm{r} 0, \mathrm{r} 1$, and $\mathrm{r} 2$ (blue).

(C) Budding decreases the total Gal1-GFP fluorescence signal in mother cells and increases the total Gal1GFP fluorescence signal in daughter cells (top). To compensate for this dilution, we summed up the total Gal1-GFP fluorescence signal of each mother cell present and its progeny during one repression (bottom).

(D) Single-cell traces of total GFP signal adjusted for dilution (see (C)) for the first two hours of repressions r1 (left) and r2 (right). Time to maximal mean total GFP is 17 min shorter in repression r2, where mean total GFP is indicated by the dotted line and the maximal mean total GFP is highlighted by the dot. Bootstrap $\left(10^{5}\right)$ samples were drawn to generate mean \pm std.

The replicate analysis can be found in Figure S1.

\section{Decreased time to maximal total GFP in repression $r 2$ at the population level}

Following a galactose-glucose shift, total GFP intensities initially rise before decreasing (Figure 2D). To determine the repression kinetics at the population level, we calculated the mean total GFP signal over time for repressions $\mathrm{r} 1$ and $\mathrm{r} 2$. Interestingly, the time to attain the maximal mean total GFP reduced from $0.79 \pm 0.10 \mathrm{~h}$ (mean \pm std, $\mathrm{n}=102$ cells) in $\mathrm{r} 1$ to $0.50 \pm 0.03 \mathrm{~h}$ ( $\mathrm{n}=328$ cells) in $\mathrm{r} 2$, where the time point of the maximal mean was bootstrapped $10^{5}$ times (Figure 2D). This demonstrates a decreased time to maximal total GFP in $\mathrm{r} 2$ at the population level. 


\section{The computational model distinguishes between repressor and non-repressor cells}

At the single-cell level, Gal1 induction delay varies significantly. Zacharioudakis et al. showed that Gal1 induction caused by a glucose-galactose media shift results in a bimodal population distribution, with only a subset of cells inducing Gal1 even after several hours of galactose exposure (16). As in our experiments, repression was preceded by $3 \mathrm{~h}$ of galactose induction, we expected that our cell population at the start of repression contained induced and uninduced cells, which show and do not show repression kinetics, respectively. However, independent of repression memory, a larger proportion of non-repressor cells in repression $r 1$ could explain the decrease in the time to maximal mean total GFP in repression $r 2$ (Figure 2D). As it is difficult to distinguish between repressor and non-repressor cells from the total GFP traces alone (Figure 2D), we used computational modeling and model selection to systematically describe the kinetics of single total GFP traces. Since Gal1 induction results in an approximate 1000-fold change in Gal1 expression (22), we assumed that stochasticity inherent to gene expression was insignificant and that a deterministic modeling approach was sufficient in describing the kinetics of the total GFP traces. To discriminate between repressor and non-repressor cells, we defined two models. The non-repressor model assumes a constant basal GFP production and degradation over time with rates $r_{\text {basal }}$ and $r_{\text {deg }}$ (Figure $3 A$ left), since we observed a gradual increase in total GFP signal in cells visually identified as not showing repression kinetics (Figure 3B top right). The temporal variation of the total GFP signal over time indicated by the non-repressor model is summarized using the following ordinary differential equation:

$$
d G F P(t) / d t=r_{\text {basal }}-r_{\text {deg }} \operatorname{GFP}(t) \text {. }
$$

This is solved by

$$
\operatorname{GFP}(t)=r_{\text {basal }} / r_{\text {deg }}\left(1-\exp \left(-r_{\text {deg }} t\right)\right)+\operatorname{GFP} P_{0} \exp \left(-r_{\text {deg }} t\right)
$$

where $\mathrm{GFP}_{0}=\mathrm{GFP}(0)$, the initial total GFP at time point 0 . According to the repressor model, cells that induced Gal1 during galactose induction required time to reestablish glucose-mediated repression. Hence, GFP is actively generated at rate $r_{\text {prod }}$ till a time point $t_{\text {delay. }}$. GFP production is switched off $\left(r_{\text {prod }}=0\right)$ and GFP is degraded with rate $r_{\text {deg }}$ after this estimated repression delay $t_{\text {delay }}$ (Figure $3 \mathrm{~A}$ right). An example of a cell visually identified as showing repression kinetics can be found in Figure 3B top left. Until $t_{\text {delay }}$, the repressor model equals the non-repressor model. The temporal change of total GFP over time described by the repressor model is summarized by the following ordinary differential equations:

$$
\begin{aligned}
& \text { before } t_{\text {delay: }}: d G F P(t) / d t=r_{\text {prod }}-r_{\text {deg }} \operatorname{GFP}(t) \\
& \text { after } t_{\text {delay }}: d G F P(t) / d t=-r_{\text {deg }} \operatorname{GFP}(t)
\end{aligned}
$$

with solutions

$$
\begin{aligned}
& \text { before } t_{\text {delay }}: \operatorname{GFP}(t)=r_{\text {prod }} / r_{\text {deg }}\left(1-\exp \left(-r_{\text {deg }} t\right)\right)+G F P_{0} \exp \left(-r_{\text {deg }} t\right) \\
& \text { after } t_{\text {delay }}: \operatorname{GFP}(t)=\operatorname{GFP}\left(t_{\text {delay }}\right) \exp \left(-r_{\text {deg }}\left(t-t_{\text {delay }}\right)\right),
\end{aligned}
$$

where $\operatorname{GFP}\left(t_{\text {delay }}\right)=r_{\text {prod }} / r_{\text {deg }}\left(1-\exp \left(-r_{\text {deg }} t_{\text {delay }}\right)\right)+G F P_{0} \exp \left(-r_{\text {deg }} t_{\text {delay }}\right)$. The non-repressor and repressor model comprise four and five model parameters, respectively: initial total GFP, GFP, basal GFP production rate, $r_{\text {basal }}$, or GFP production rate, $r_{\text {prod, }}$ GFP degradation rate $r_{\text {deg, }}$, a noise parameter $\sigma$ determining the width of the Gaussian noise distribution (see Materials and 
Methods), and the repression delay $t_{\text {delay }}$ for the repressor model. For repressions $r 1$ and $r 2$, respectively, we performed multi-start maximum likelihood optimization and model selection on both models for each total GFP trace (Figure 3B top and $3 \mathrm{C}$ ). Calculating the profile likelihoods of exemplary total GFP traces, we found the model parameters of the repressor model to be identifiable (Figure 3B bottom). We then determined whether active repression, i.e. the repressor model, was required to explain a total GFP trace using the Bayesian information criterion (BIC). $A B I C$ difference of ten between the repressor and non-repressor model $\left(\mathrm{BIC}_{\text {repressor }}<\mathrm{BIC}_{\text {non- }}\right.$ repressor - 10) was considered an appropriate threshold to reject the non-repressor model with fewer model parameters (see Materials and Methods and Figure 3B). Of all total GFP traces, $71 \%$ and $76 \%$ of $r 1$ and $r 2$, respectively, were discovered to require the repressor model (Figure 3D) and are henceforth referred to as "repressor cells." The higher proportion of repressor cells was expected due to Gal1 transcriptional reinduction memory resulting in increased proportions of cells producing detectable GFP in induction i2. The median initial total GFP, GFP , was significantly higher in repressor cells than in non-repressor cells $\left(p=9.2 \cdot 10^{-5}\right.$ for $\mathrm{r} 1$ and $p=$ $2.7 \cdot 10^{-7}$ for $r 2$ ) (Figure 3D). This implies that our repression models can discriminate between cells that were repressing Gal1 and cells uninduced at the beginning of repression. The overlapping ranges of initial total GFP between repressor and non-repressor cells reveal how simple thresholding could result in wrong differentiation between repressor and non-repressor cells.

\section{Decreased time to maximal total GFP is also present in the repressor cell subpopulation}

To determine whether the previously described decrease in time to maximal mean total GFP in r2 is due to a different fraction of non-repressor cells between $r 1$ and $r 2$, we computed the times to maximal mean total GFP on the repressor cell subpopulation. We again found a shortened time to maximal mean total GFP in $r 2$, with $0.81 \pm 0.09$ (mean \pm std, $n=72$ cells) and $0.52 \pm 0.05$ ( $n=$ 248 cells) $h$ for $r 1$ and $r 2$, respectively (Figure $3 E$ ), demonstrating that the decrease in time to maximal total GFP in $r 2$ is not due to a different fraction of non-repressor cells.

\section{Shortened repression delay in repression $r 2$ at the single-cell level}

To address if the repression kinetics are different between $r 1$ and $r 2$ in individual cells, we compared the paired estimated single-cell parameters of repressor cells present in both repressions. We discovered that the median initial total GFP, GFP , and median repression delay, $t_{\text {delay, }}$ are substantially different $\left(p=3.3 \cdot 10^{-9}\right.$ and $p=1.5 \cdot 10^{-3}$, respectively) between both repressions using a two-sided paired sign test and multiple test correction (Figure 3F). Median GFP $_{0}$ is increased (median values of 0.59 and 2.05 for $r 1$ and $r 2$, respectively), while median $t_{\text {delay }}$ is shortened in $\mathrm{r} 2$ (median values of 0.50 and 0.38 for $r 1$ and $r 2$, respectively, where $72 \%$ of paired cells showed a decrease in $t_{\text {delay }}$ ), in line with the previously identified decrease in the time to the maximal mean total GFP in $r 2$ at the repressor subpopulation level (Figure 3E). The increased GFP $_{0}$ in $\mathrm{r} 2$ conforms to the transcriptional reinduction memory of Gal1 and results in higher GFP 0 at the start of $r 2$. The median $r_{\text {prod }}$ and median $r_{\text {deg }}$ between the two repressions were comparable 
( $p=0.22$ and $p=0.34$, respectively) (Figure 3F). In line with our findings, Bheda et al. also identified similar production rates (17). We repeated the entire wildtype analysis based on data from independent experiments (see Methods for details). This replicate analysis confirms the conclusions presented in Figures $2 \mathrm{~B}, 2 \mathrm{D}$ and 3 , in particular the earlier repression response in repression r2 (Figure S1).
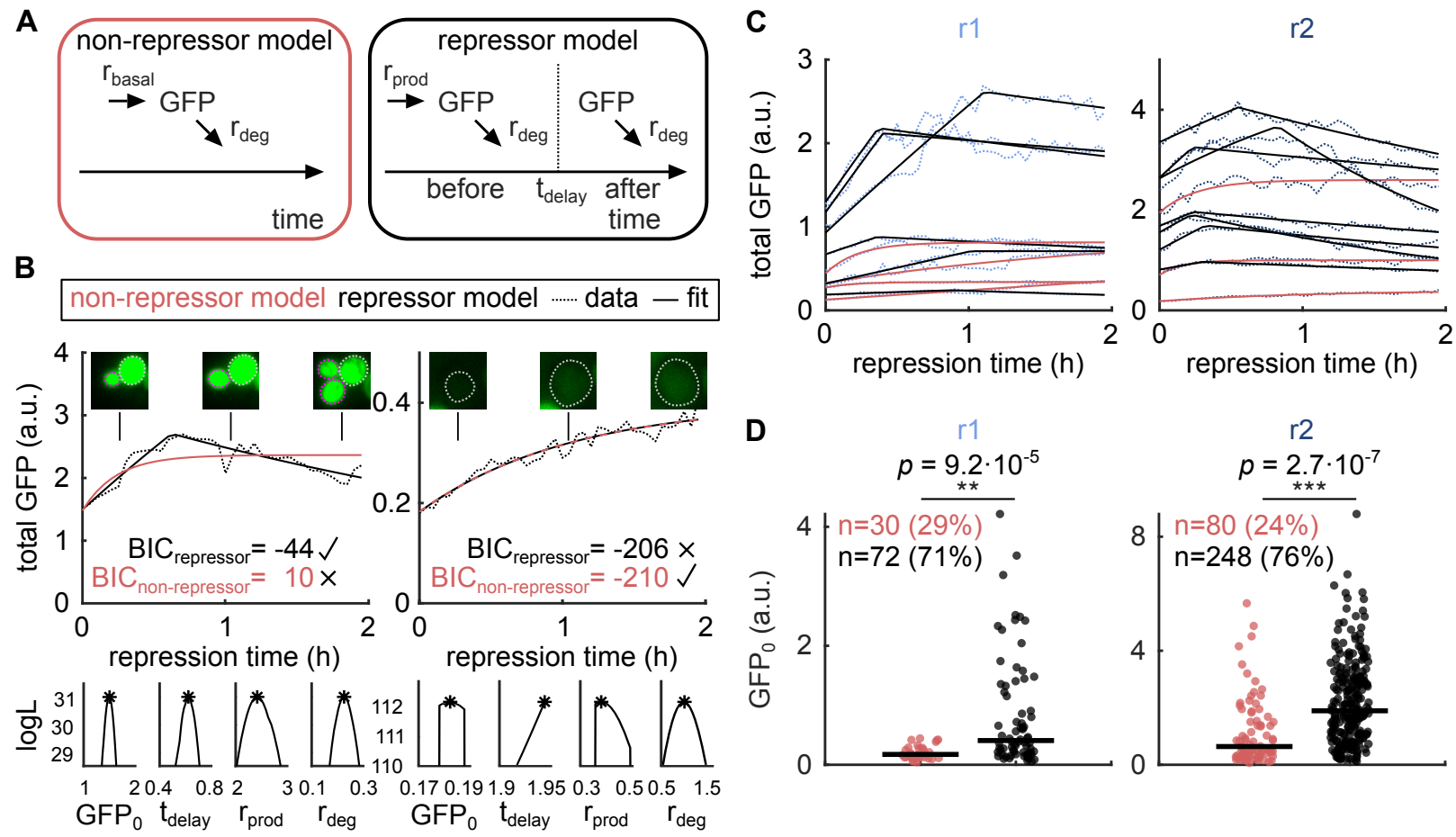

E

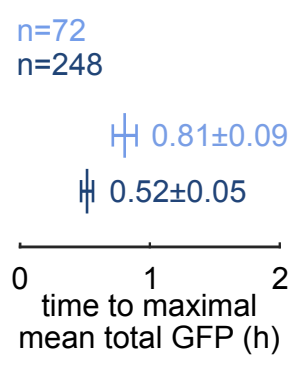

$\mathbf{F}$
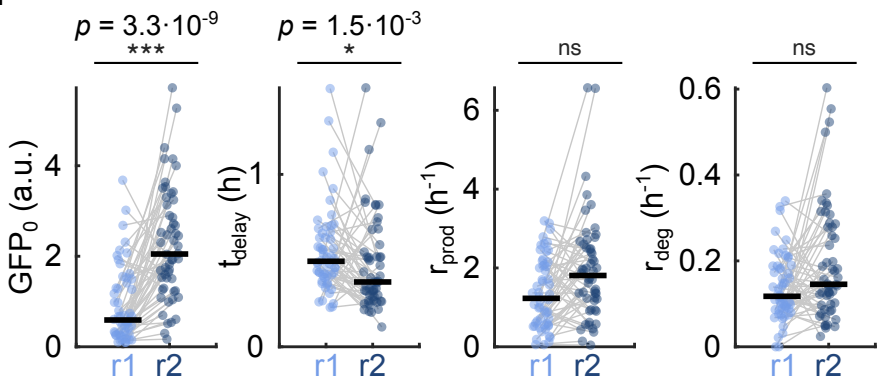

Figure 3. Shortened repression delay in repression $r 2$ at the single-cell level.

(A) Left: a model for non-repressor cells composed of basal GFP production ( $\left.r_{\text {basal }}\right)$ and degradation $\left(r_{\text {deg }}\right)$. Right: a model for repressor cells composed of an initial constant and active GFP production ( $r_{\text {prod }}$ ) and degradation $\left(r_{\text {deg }}\right)$ until a delayed repression onset $\left(t_{\text {delay }}\right)$ where GFP production is switched off.

(B) Top: two exemplary total GFP traces (dotted line) and fits of the non-repressor model (red solid line) and repressor model (black solid line). Exemplary images of the cell(s) at three different time points are shown above. Mother cells are circled in gray, progeny in pink. The better fitting model was selected according to the Bayesian information criterion (BIC). Left: total GFP trace better fitted by the repressor model. Right: total GFP trace fitted equally well by the non-repressor and repressor model. Due to the higher model complexity of the repressor model, the repressor model is still rejected. Bottom: profile 
likelihoods of the repressor model corresponding to the two exemplary total GFP traces above endorse parameter identifiability. Asterisks represent optimized parameters and corresponding log-likelihood (logL) values.

(C) Ten exemplary total GFP traces (dotted lines) and best fits (solid lines) for repressions $\mathrm{r} 1$ (left) and $\mathrm{r} 2$ (right). GFP traces best fitted with a repressor model are shown in black and fits of total GFP traces best fitted with a non-repressor model are shown in red.

(D) The median initial total GFP, GFPo, is significantly higher $\left(p=9.2 \cdot 10^{-5}\right.$ and $p=2.7 \cdot 10^{-7}$, two-sided median test corrected for multiple testing with Bonferroni correction, $m=20$ ) in traces better fitted by the repressor model (black) than in traces better fitted by the non-repressor model (red). This confirms that the repressor model fits induced cells better, while the non-repressor model fits uninduced cells. The number of cells and percentages of all GFP traces best fitted by the repressor model and non-repressor model are shown.

(E) Time to maximal mean total GFP is decreased in repression $r 2$ for repressor cells $(0.81 \pm 0.09$ vs. 0.52 $\pm 0.05)$. Bootstrap $\left(10^{5}\right)$ samples of the repressor cells were drawn to generate mean \pm std.

(F) Comparison of paired estimated single-cell parameters of repression $\mathrm{r} 1$ and $\mathrm{r} 2$ shows that the median initial total GFP, GFP, and median repression delay, tdelay, are significantly different $\left(p=3.3 \cdot 10^{-9}\right.$ and $p=$ $1.5 \cdot 10^{-3}$, respectively, two-sided paired sign test correcting for multiple testing with Bonferroni correction,

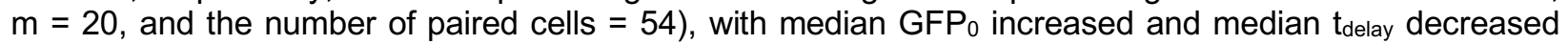
(median $t_{\text {delay }}$ values of 0.50 and 0.38 for $\mathrm{r} 1$ and $\mathrm{r} 2$, respectively). Median production rate, $\mathrm{r}_{\text {prod, }}$ and median degradation rate, $r_{d e g}$, are not significantly different between $\mathrm{r} 1$ and $\mathrm{r} 2(p=0.22$ and $p=0.34$, respectively). The replicate analysis can be found in Figure $\mathrm{S} 1$.

\section{Earlier repression response in repression $r 2$ for elp6 $\Delta$ cells}

Intrigued by the findings in wildtype yeast cells, we repeated our analysis for the previously identified gain-of-reinduction memory mutant Elp6 (elp6 $\Delta)$. The total Gal1-GFP fluorescence signal of single elp6 6 cells demonstrates Gal1 induction and repression during galactose and glucose and reinduction memory (Figure 4A). We performed dilution compensation (Figure 2C) on repressions $\mathrm{r} 1$ and $\mathrm{r} 2$ (Figure $4 \mathrm{~B}$ ) and calculated the mean total GFP signals over time to determine the repression kinetics at the population level. Similar to wildtype cells, we discovered that the time to attain the maximal mean total GFP decreased from $1.40 \pm 0.09 \mathrm{~h}$ ( $\mathrm{n}=66$ cells) in $r 1$ to $0.71 \pm 0.07 \mathrm{~h}$ ( $\mathrm{n}=237$ cells) in $\mathrm{r}$, (Figure 4B). Subsequently, we repeated multi-start maximum likelihood optimization and model selection for the non-repressor and repressor models to show that the shortened time to maximal mean total GFP in r2 is not caused by a larger proportion of non-repressor cells in $\mathrm{r} 1$ (Figure $4 \mathrm{C}$ ). Of all total GFP traces, $64 \%$ and $83 \%$ of elp6 cells in $\mathrm{r} 1$ and $\mathrm{r} 2$ require the repressor model (Figure 4D). When determining the time to maximal mean total GFP for the elp6 $\Delta$ repressor subpopulation, we again identified a shortened time to maximal mean total GFP, with $1.20 \pm 0.14$ ( $n=42$ cells) and $0.69 \pm 0.07$ ( $n=196$ cells) $h$ for $r 1$ and $\mathrm{r} 2$, respectively (Figure $4 \mathrm{E}$ ). To investigate the earlier repression response at the single-cell level, we compared the paired estimated single-cell parameters of elp6 $\Delta$ repressor cells present in both repressions. We found the median $\mathrm{GFP}_{0}$, and median $\mathrm{t}_{\text {delay }}$, to be significantly different $(p$ $=1.2 \cdot 10^{-10}$ and $p=3.9 \cdot 10^{-5}$ ) between repressions (Figure $4 \mathrm{~F}$ ). Similar to wildtype cells, the median GFP $_{0}$ increased (median values of 0.57 and 3.69 for $\mathrm{r} 1$ and $\mathrm{r} 2$, respectively), while the median $t_{\text {delay }}$ is shortened in $r 2$ (median values of 0.95 and 0.50 for $r 1$ and $r 2$, respectively, where $85 \%$ of paired cells showed a decrease in $t_{\text {delay }}$ ), as previously identified at the repressor subpopulation level. The increased initial total GFP level in r2 was expected due to the previously identified reinduction memory of elp6 $\Delta$ cells. Contrary to wildtype cells, we discovered that for 
elp6 6 cells the median production rate, $r_{\text {prod, }}$ is significantly different between $r 1$ and $r 2(p=$ $6.9 \cdot 1^{-8}$ ) with increased median $r_{\text {prod }}$ in $r 2$ (median values of 1.10 and 3.10 for $r 1$ and $r 2$, respectively, Figure 4F) reflecting the gain-of-reinduction-memory phenotype reported by Bheda et al. (17). Median $r_{\text {deg }}$ was comparable between repressions $(p=0.39)$. We repeated the elp6 $\Delta$ analysis based on additional data from independent experiments (see Methods for details). The replicate analysis confirms the conclusions presented in Figure 4, in particular the earlier repression response in repression $\mathrm{r} 2$ (Figure S2).

A

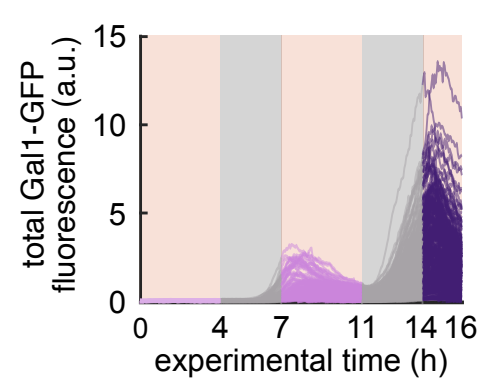

B

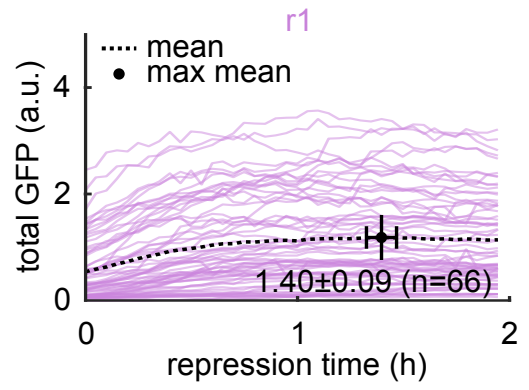

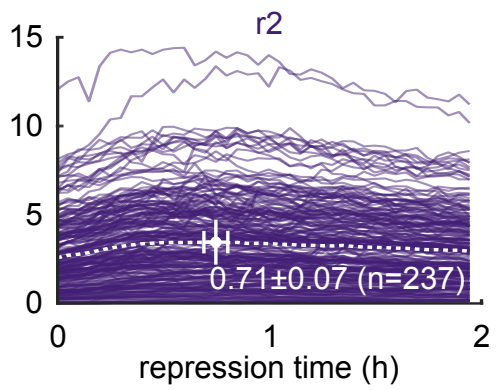

repression time $(\mathrm{h})$
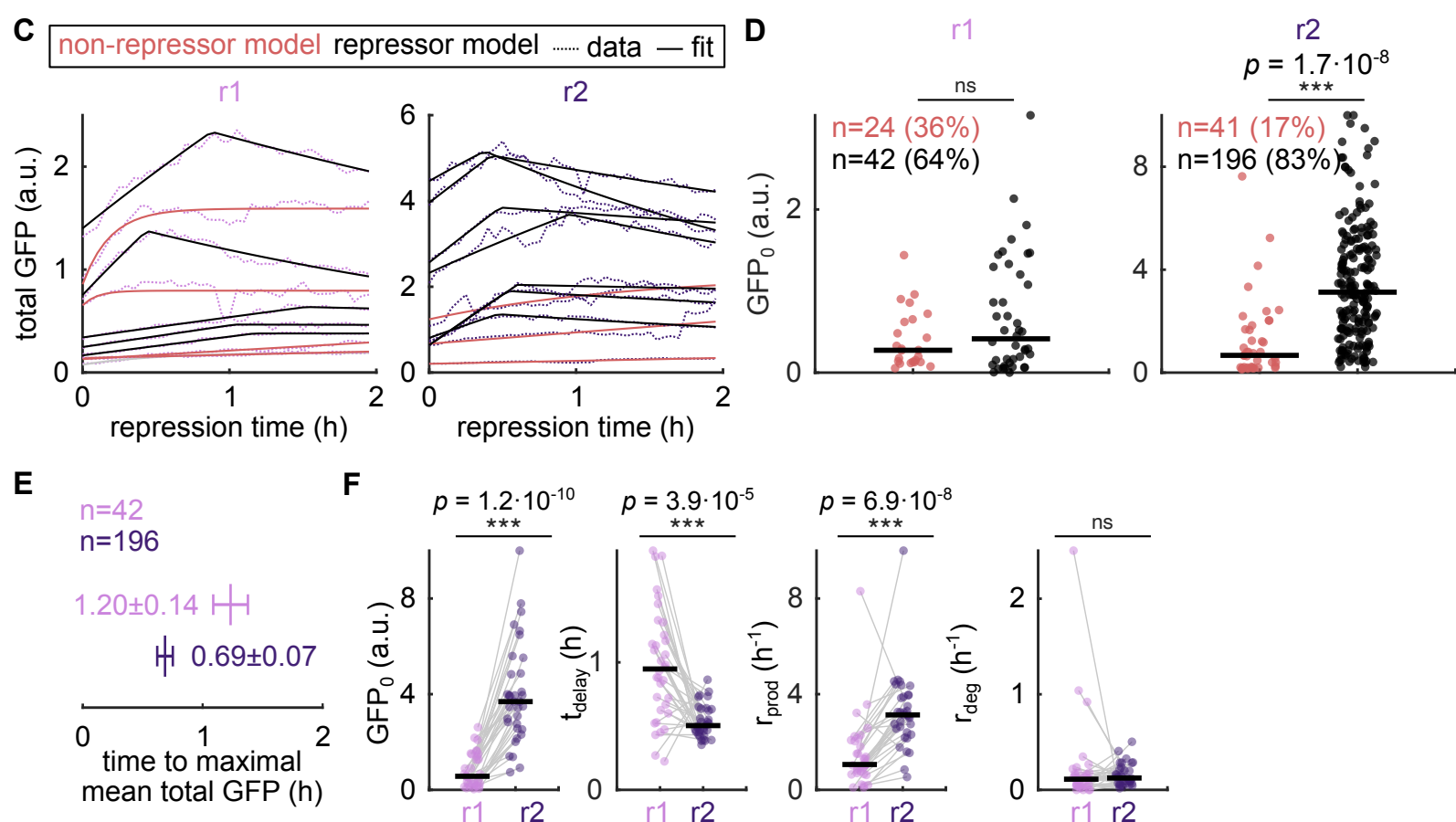

$\mathrm{F}$

repression time $(\mathrm{h})$
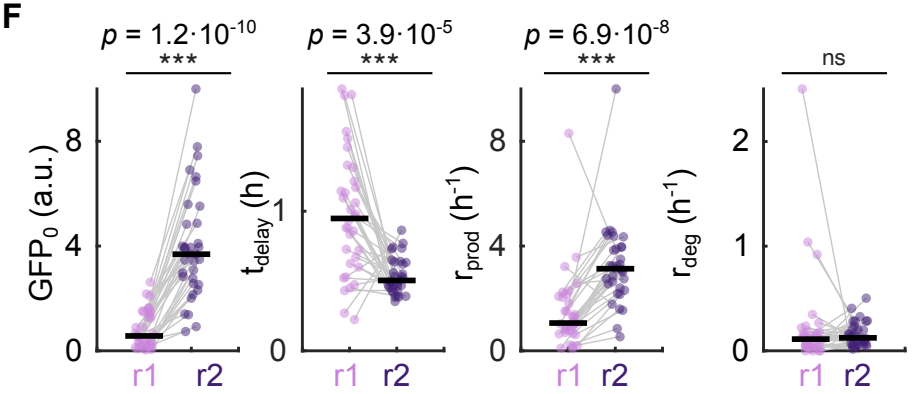

Figure 4. Earlier repression response in repression $r 2$ for elp6 cells at both the population and single-cell level.

(A) Single-cell traces of total Gal1-GFP fluorescence signal of elp6 budding yeast cells across two inductions i1 and i2 (gray) and repressions 0, 1, and 2 (purple).

(B) Single-cell traces of total GFP signal of elp6 $\Delta$ budding yeast cells adjusted for dilution (see Figure 2C) for the first two hours of repression 1 ( $r 1$, left) and repression 2 ( $r 2$, right). Time to maximal mean total GFP is $41 \mathrm{~min}$ shorter in repression $\mathrm{r}$, where the mean total GFP signal is indicated by the dotted line and the maximal mean total GFP is highlighted by the dot. Bootstrap $\left(10^{5}\right)$ samples were drawn to generate mean \pm std. 
(C) Ten exemplary total GFP traces (dotted lines) and best fits (solid lines) of elp6 $\Delta$ budding yeast cells and repressions $\mathrm{r} 1$ (left) and $\mathrm{r} 2$ (right).

(D) The median initial total GFP, GFP , is higher in elp6 $\Delta$ traces better fitted by the repressor model (black) than in elp6 $\Delta$ traces better fitted by the non-repressor model (red). This confirms that the repressor model fits induced elp6 $\Delta$ cells better, while the non-repressor model fits uninduced elp6 $\Delta$ cells. The number of cells and percentages of all GFP traces best fitted by the repressor model and non-repressor model are shown in brackets.

(E) Time to maximal mean total GFP is decreased in repression $r 2$ for elp6 $\Delta$ repressor cells $(1.20 \pm 0.14$ vs. $0.69 \pm 0.07)$. Bootstrap $\left(10^{5}\right)$ samples of the repressor cells were drawn to generate mean \pm std.

(F) Comparison of paired estimated single-cell parameters of elp6 $\Delta$ cells of repression $\mathrm{r} 1$ and $\mathrm{r} 2$ show that median initial total GFP, GFP, median repression delay, tdelay, and median production rate, $r_{\text {prod, }}$ are significantly different $\left(p=1.2 \cdot 10^{-10}, p=3.9 \cdot 10^{-5}\right.$ and $p=6.9 \cdot 10^{-8}$, respectively, two-sided paired sign test correcting for multiple testing with Bonferroni correction, $m=20$, and the number of paired cells is 34), with both median GFP $_{0}$ and median $r_{\text {prod }}$ increased and median tdelay decreased (median tdelay values of 0.95 and $0.50 \mathrm{~h}$ for $\mathrm{r} 1$ and $\mathrm{r} 2$, respectively) in $\mathrm{r} 2$. Median degradation rate, $\mathrm{r}$ deg, is not significantly different between $\mathrm{r} 1$ and $\mathrm{r} 2(p=0.39)$.

The elp $6 \Delta$ replicate analysis can be found in Figure S2.

\section{elp6 $\Delta$ shows stronger repression delay impairment in first repression}

To first identify if the repression kinetics between wildtype and elp6 $\Delta$ cells differed at the repressor subpopulation level, we compared the repression kinetics of wildtype and elp6 $\Delta$ repressor cells. We found that the time to maximal mean total GFP increased for elp6 $\Delta$ repressor cells for repression $r 1$ (Figure 5A). Next, we wanted to identify if the elp6 $\Delta$ repressor cells show altered repression kinetics at the single-cell level compared to wildtype cells. As we here assess different strains, we were no longer able to pair cells and, thus, compared the estimated single-cell parameters of all repressor cells. Using a two-sided median test and correcting for multiple testing, we discovered that the median $t_{\text {delay }}$ for wildtype was significantly lower (median $t_{\text {delay }}=0.52 \mathrm{~h}$ ) compared to elp6 $\Delta$ (median $t_{\text {delay }}=0.88 \mathrm{~h}, p=4.7 \cdot 10^{-4}$ ) for $\mathrm{r} 1$ (Figure 5B). However, median $\mathrm{GFP}_{0}$, median $r_{\text {prod, }}$ and median $r_{\text {deg }}$ are comparable $(p=1, p=1$, and $p=0.44$, respectively) (Figure $5 \mathrm{~B})$. This is in line with previous findings that wildtype and elp6 cells have similar Gal1 levels in induction i1 leading to comparable median GFP $_{0}$ in $\mathrm{r} 1$ and comparable production rates between wildtype and elp6 6 cells during induction i1 (17). Then, we performed the same analysis for repression r2. For $\mathrm{r} 2$, we found that the time to maximal mean total GFP at the repressor subpopulation level was more similar between wildtype and elp6 $\Delta$ repressor cells (Figure $5 \mathrm{C}$ ) than in $r 1$. At the single-cell level, the median $\mathrm{GFP}_{0}$, median $t_{\text {delay, }}$ and median $r_{\text {prod }}$ were significantly different $\left(p=1.8 \cdot 10^{-6}, p=2.2 \cdot 10^{-3}\right.$, and $p=8.1 \cdot 10^{-11}$, respectively) between wildtype and elp6 $\Delta$ cells for $\mathrm{r} 2$ (Figure 5D) (see Materials and Methods), whereas median $r_{\text {deg }}$ was comparable after correcting for multiple testing $(p=0.04)$ (see Materials and Methods). Overall, this analysis identifies a - compared to wildtype - stronger repression delay impairment of elp6 $\Delta$ cells in $\mathrm{r} 1$, while the repression delay in $\mathrm{r} 2$ is more similar to wildtype cells. The previously described gain-of-induction-memory phenotype of elp6 6 , which showed identical Gal1 levels to wildtype in induction i1 but increased Gal1 levels in induction i2, is responsible for the increased median GFP $_{0}$ (17). Furthermore, Bheda et al. reported increased elp6 $\Delta$ production rates for induction i2 but not for induction i1. 


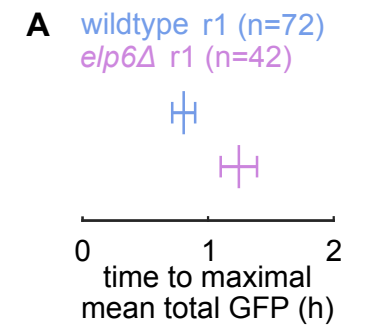

C wildtype $\mathrm{r} 2(\mathrm{n}=248)$ D elp6 $\mathrm{r} 2(\mathrm{n}=196)$

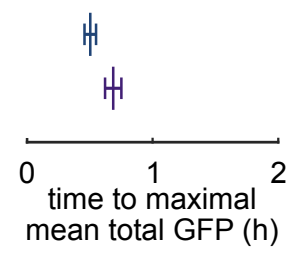

B

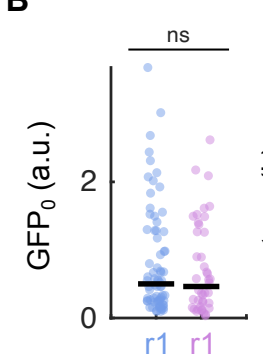

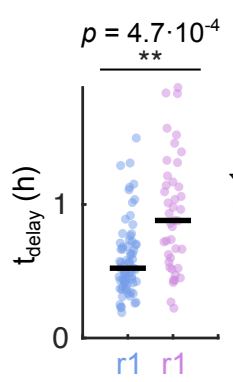
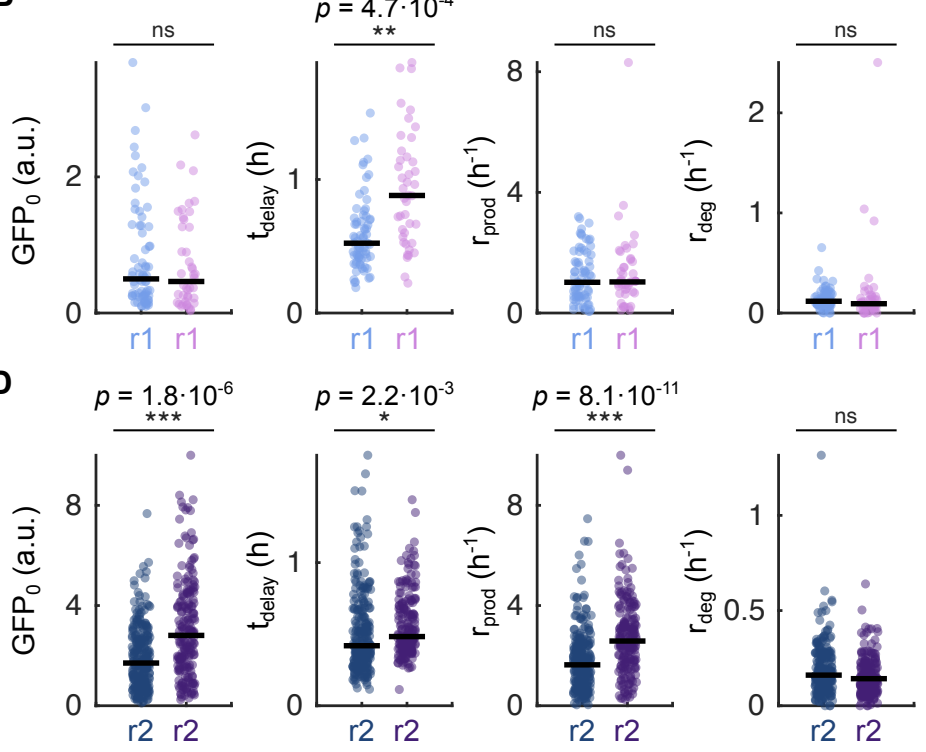

Figure 5. Stronger Gal1 repression delay impairment in elp6 $\Delta$ cells during first repression.

(A) Time to maximal mean total GFP is increased for elp6 $\Delta$ repressor cells for repression $r 1(0.81 \pm 0.09$ vs. $1.20 \pm 0.14)$. Bootstrap $\left(10^{5}\right)$ samples were drawn to generate mean \pm std.

(B) Comparison of estimated single-cell parameters of wildtype and elp6 cells of repression $r 1$ shows that elp6 $\Delta$ has a significantly different median repression delay, $t_{\text {delay }}$, in comparison to wildtype, $\left(p=4.7 \cdot 10^{-4}\right.$, two-sided median test correcting for multiple testing with Bonferroni correction, $\mathrm{m}=20$, and the number of cells for wildtype and elp6 6 is 72 and 42 , respectively) with median tdelay increased in elp $6 \Delta$ (median values of 0.52 and $0.88 \mathrm{~h}$ for wildtype and elp6 , respectively). Median initial total GFP, GFP, median production rate, $r_{\text {prod, }}$ and median degradation rate, $r_{\text {deg }}$, are not significantly different between wildtype and elp6 $\Delta$ cells of repression $\mathrm{r} 1$ ( $p=1, p=1$, and $p=0.44$, respectively).

(C) Time to maximal mean total GFP is more comparable for wildtype and elp6 repressor cells for repression $\mathrm{r} 2(0.52 \pm 0.05$ vs. $0.69 \pm 0.07)$. Bootstrap $\left(10^{5}\right)$ samples were drawn to generate mean \pm std.

(D) Comparison of estimated single-cell parameters of wildtype and elp6 $\Delta$ cells of repression 22 shows median GFP 0 , median tdelay, and median $r_{\text {prod, }}$, to be significantly different in elp $6 \Delta$ compared to wildtype $(p$ $=1.8 \cdot 10^{-6}, p=2.2 \cdot 10^{-3}$, and $p=8.1 \cdot 10^{-11}$, respectively, two-sided median test correcting for multiple testing with Bonferroni correction, $m=20$ and number of cells for wildtype and elp6 $\Delta$ is 248 and 196, respectively), with median $\mathrm{GFP}_{0}$, median $\mathrm{t}_{\text {delay }}$, and median $\mathrm{r}_{\text {prod }}$ increased in elp6 $\Delta$ (median values of 0.42 and $0.48 \mathrm{~h}$ for wildtype and elp6 6 , respectively). Median $r_{\text {deg }}$ is not significantly different between wildtype and elp6 $\Delta$ cells of repression $\mathrm{r} 2(p=0.04)$ after correcting for multiple testing.

\section{DISCUSSION}

Based on population data, we found an earlier repression response upon a repeated repression. We used mathematical modeling to demonstrate that changes in the repression response were not simply due to different proportions of repressor cells. Interestingly, we discovered that elp6 $\Delta$ cells showed prolonged repression delay in the first repression but a more comparable delay in comparison to wildtype in the second repression.

\section{Repression memory manifests as faster initiation of second repression}

At both the population and single-cell level, we identified that both wildtype and elp6 $\Delta$ cells have an earlier repression response in repression r2 (Figures 3E-F, 4E-F, S1E-F, S2E-F). This implies 
that cells repeatedly exposed to glucose are faster at initiating Gal1 repression. We thus propose that budding yeast cells do not only show reinduction memory when exposed to repeated galactose inductions (17) but also have a repression memory when exposed to repeated glucose repressions. We hypothesize that faster repression of the galactose-metabolizing machinery would save energy and/or allow for faster induction of the glucose-metabolizing machinery, which would be beneficial to individual yeast cells. It will be intriguing to investigate how Gal1 repression is acquired on a molecular level and if glucose and galactose repression memories are linked in the future. We would like to note here that we assessed the kinetics of the Gal1-GFP fusion protein, which has been successfully employed in several studies involving Gal1 gene expression $(16,23-26)$, and hence also modeled Gal1-GFP kinetics. Moreover, it should be mentioned that potential photo-bleaching effects would not influence the estimated repression delays, and, thus, our repression memory hypothesis. When considering memory as the fold change of the second delay to the first delay, we discover reinduction memory to have an overall stronger effect size. The fold changes of the repression delays are (median $t_{\text {delay }} r 2 /$ median $t_{\text {delay }} r 1=0.38 / 0.50=0.76$ ) 0.76 for wildtype and (median $t_{\text {delay }} r 2 /$ median $t_{\text {delay }} r 1=0.50 / 0.95=0.53$ ) 0.53 for elp6 . In comparison, the fold changes of the induction delays are (median $t_{\text {delay }} \mathrm{i} 2 /$ median $t_{\text {delay }} \mathrm{i} 1=$ $0.67 / 2.33=0.29$ ) 0.29 and (median $t_{\text {delay }} \mathrm{i} 2 /$ median $t_{\text {delay }} \mathrm{i} 1=0.50 / 2.50=0.20$ ) 0.20 for wildtype and elp6 $\Delta$, respectively, as reported in Bheda et al. (17).

\section{elp6 $\Delta$ is a novel gain-of-repression-memory mutant}

We observed that elp $6 \Delta$ cells had longer repression delay in repression $\mathrm{r} 1$ than wildtype cells, while the repression delay is more comparable for wildtype and elp6 $\Delta$ cells in repression $\mathrm{r} 2$ at both the population and single-cell level (Figure 5). This reveals that the repression memory effects (shortening of repression delay in $\mathrm{r} 2$ ) of elp6 $\Delta$ cells are stronger than in wildtype cells. Hence, elp6 $\Delta$ can be classified as a gain-of-repression-memory mutant. Elp6 has been identified as one of the six subunits of the so called RNA polymerase II elongator complex (24) and has been linked to a variety of biological functions (25-27). However, its precise function is still debated. Contrary to induction, where elp6 $\Delta$ and wildtype show comparable induction delays in the first induction but exhibit significantly different induction delays in the second induction, elp6 $\Delta$ and wildtype cells show significantly different repression delays in $\mathrm{r} 1$ and more comparable repression delays in $\mathrm{r} 2(17)$ (Figure 5B and $\mathrm{D}$ ). Although reinduction memory has a stronger overall effect size than repression memory, the relative memory effect of elp6 $\Delta$ and wildtype is comparable between induction (elp6 $\Delta$ induction memory/wildtype induction memory $=0.20 / 0.29$ $=0.69$ ) and repression (elp6 $\Delta$ repression memory/wildtype repression memory $=0.53 / 0.76=$ $0.70)$. Whether the gain-of-repression memory is a unique characteristic of elp6 $\Delta$ cells or if other knock-out mutants show similar repression kinetics and delay responses will be interesting to investigate in the future. 


\section{ACKNOWLEDGMENTS}

L.S. was funded by the BMBF project TIDY (031L0170B). L.S. is especially grateful to the Technical University of Munich's Department of Mathematics, whose Entrepreneurial Award (within the Program "Global Challenges for Women in Math Science") contributed to the completion of this project. L.S. acknowledges further support by the Add-on Fellowship for Interdisciplinary Life Science of the Joachim Herz Foundation. C.M. acknowledges funding from the European Research Council (ERC) under the European Union's Horizon 2020 research and innovation program (grant agreement no. 866411). The work in R.S. laboratory was supported by the Deutsche Forschungsgemeinschaft (DFG, German Research Foundation) through SFB 1064 (Project-ID 213249687) and SFB 1309 (Project-ID 325871075), the AmPro program (ZT0026) and Helmholtz Gesellschaft.

\section{AUTHOR CONTRIBUTIONS}

Conceptualization, P.B., R.S. and C.M.; Methodology, L.S.; Software, L.S.; Validation, L.S.; Formal Analysis, L.S.; Investigation, L.S., I.K.; Resources, R.S., and C.M.; Data Curation, L.S., M.B.; Writing - Original Draft, L.S.; Writing - Review \& Editing, L.S., M.S., R.S. and C.M.; Visualization, L.S.; Supervision, P.B., R.S., and C.M.; Project Administration, R.S. and C.M.; Funding Acquisition, R.S., and C.M..

\section{DECLARATION OF INTERESTS}

The authors declare no conflict of interests. 


\section{MATERIALS AND METHODS}

\section{Data acquisition and sources}

For the analysis for Figures 1-5, we used microscopy images and initial segmentation, mapping, and tracking information from a microfluidics experiment from Bheda et al. (17), which contained 13 and ten positions for wildtype and elp6 , respectively. Positions were chosen according to the feasibility of analysis with respect to cell movement, number and morphology, where each position represents a biological replicate. The images from the first two hours of repression $r 1$ were rectified, and the segmentation, mapping, and tracking were extended to the entire two hours of repression $r 2$. Bheda et al. only segmented $r 2$ partially since they were primarily interested in galactose induction, and did not adjust the final repression frames. Using the software PhyloCell (19), we manually corrected the segmentation, mapping, and tracking of $\mathrm{r} 1$ and $\mathrm{r} 2$ for wildtype and elp6 $\Delta$. For the technical replicate analysis (Figures S1 and S2) we repeated the inductionrepression experiment as described in Bheda et al. (17) (Figure 2A). Due to low cell numbers, we pooled data from three and two independent experiments for wildtype and elp6 $\Delta$, totaling 13 and 23 positions, respectively. Using the software YeaZ (20) and Cell-ACDC (21) for cell segmentation, mapping and tracking, we extracted the relevant single-cell information of the livecell images for both repressions $\mathrm{r} 1$ and $\mathrm{r} 2$. During glucose repression, the yeast cells proliferated, increasing the cell numbers within the microfluidic chambers. However, filled microfluidic chambers no longer assure that all the progeny of a cell is recorded, and mapping and tracking of cells become infeasible. To ensure mapping and tracking of single yeast cells within the microfluidic chambers, the glucose repressions were limited to a maximum of $4 \mathrm{~h}$ and the overall experiment was limited to $16 \mathrm{~h}$ ( $4 \mathrm{~h}$ in glucose (r0), $3 \mathrm{~h}$ in galactose (i1), $4 \mathrm{~h}$ in glucose ( $\mathrm{r} 1), 3 \mathrm{~h}$ in galactose (i2), $2 \mathrm{~h}$ in glucose (r2)).

\section{Data preprocessing}

We extracted the single-cell information relevant for our analysis, namely cell ID, mother cell ID, detection frame (first frame in which a cell is detected), last frame (last frame a cell is detected), relative GFP intensities per time (mean GFP intensity of a segmented cell) and cell area per time. As the data regarding the relative GFP intensities and cell area was not sorted over time, we first sorted it and then calculated the total GFP fluorescence per time given by

total GFP fluorescence $=$ relative GFP fluorescence $\times$ cell area.

Finally, cells that were not imaged till the end of the experiment, cells with missing relative GFP and/or cell area values, and cells that were supposedly detected before their mother cells (segmentation error) were discarded.

\section{Dilution compensation}

Cytoplasmic proteins are disseminated between the mother and daughter cells during budding. Assuming that Gal1 is not produced or degraded, protein redistribution causes a drop in total Gal1-GFP fluorescence in the mother cell and a rise in total Gal1-GFP fluorescence in the daughter cell till the mother and daughter cells split (Figure $2 \mathrm{C}$ top). As a result, regardless of 
repression, dilution causes variations in total Gal1-GFP fluorescence. The daughter cell grows to about $1 / 3$ of the size of the mother cell (27) such that the decrease in total Gal1-GFP fluorescence due to dilution was expected to be $1 / 3$ of the initial total Gal1-GFP fluorescence of the mother cell. To ensure that dilution does not overshadow potentially more subtle repression kinetics, we created artificial non-dividing cells compensating for dilution by adding the total Gal1-GFP fluorescence of the progeny of a cell present at the start of glucose repression, which we called mother cell, to the total Gal1-GFP fluorescence of that mother cell during the first $2 \mathrm{~h}$ of repression (Figures 2C bottom, 2D, 4B, S1B, and S2B). For mother cells with a bud at the beginning of a repression period, we additionally added the bud to the total Gal1-GFP fluorescence of that mother cell. The GFP traces of all computed non-dividing cells can be found under https://github.com/marrlab/Gal1repression. As we found the maximal mean total GFP to be attained before $2 \mathrm{~h}$ of glucose exposure, we restricted our analysis to the first $2 \mathrm{~h}$ of repression.

\section{Models}

During the first two hours of glucose repression, we modeled the kinetics of the total GFP of every single cell. Due to the high variability in galactose induction, we assumed that our initial cell population at the beginning of repression contained induced and uninduced cells, which show and do not show repression kinetics, respectively. We developed two models, the repressor and the non-repressor model, to account for both total GFP kinetics during repression.

\section{Non-repressor model}

For more information regarding the non-repressor model, see the main text.

\section{Repressor model}

For more information regarding the repressor model, see the main text.

\section{Noise model}

Experimental data, such as total GFP per cell per time, is noise corrupted. As a result, we used an underlying additive Gaussian noise model with a constant variance $\sigma^{2}$ throughout time to test our models. The single-cell specific model parameters are comprised in the parameter vector $\Theta_{i}$ for cell $i$ and the experimental measurement at time point $k$ for cell $i$ is denoted by $\underline{y}_{i}{ }^{k}$. The loglikelihood for the Gaussian noise model is given by

$$
\log L\left(\Theta_{i}\right)=-1 / 2 \sum_{k}\left(\log \left(2 \pi \sigma_{i}^{2}\right)+\left(y_{i}^{k}-y\left(t_{k}, \Theta_{i}\right)\right)^{2} / \sigma_{i}{ }^{2}\right) \text {. }
$$

We obtained the optimal model parameters of both models for the total GFP traces for each cell by performing maximum likelihood estimation.

\section{Optimization and parameter estimation}

For each total GFP trace separately for $r 1$ and $r 2$, wildtype, and elp6 6 , we computed the model

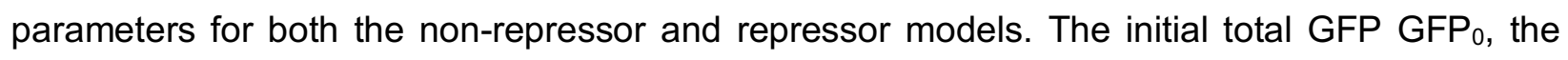


basal production rate $r_{\text {basal, }}$ and the degradation rate $r_{\text {deg }}$ are the model parameters for the nonrepressor model. Instead of a basal production rate, $r_{\text {basal, }}$, we have a production rate, $r_{\text {prod, }}$, for the repressor model. Also, we discovered the time point of delayed repression $t_{\text {delay }}$ for the repressor model. For both models we also infer one noise parameter $\sigma$ determining the spread of the Gaussian noise model. We assumed that all parameters are constant over time. For numerical reasons we optimized the parameters in $\log _{10}$ scale (28) and rescaled the data by $10^{7}$. As total Gal1-GFP fluorescence signal and total Gal1-GFP molecules are (linearly) mapped by an unknown constant, the number of total Gal1-GFP molecules is always scalable by that unknown constant that we exploit to increase convergence. The lower and upper bounds for all initial, rate, and noise parameters are -10 and 1 in $\log _{10}$ scale, respectively, assuring that the whole range of biologically plausible parameter values is covered. The lower and upper bounds for the repression delay are given by $36 \mathrm{~s}$ and $2 \mathrm{~h}$ (corresponding to -2 and $\log _{10}(2)$ in $\log _{10}$ scale). As we only considered $2 \mathrm{~h}$ of glucose repression, we did not allow the time delay to take on larger values. We performed multi-start local optimization of the negative log-likelihood using the parameter estimation toolbox PESTO (29). For each model and total GFP trace, we performed local optimization runs from at least 20 different Latin-hypercube-sampled starts. If less than five starts converged, i.e. the objective function values of the starts differ less than 0.1 to the best start, we re-ran the optimization with 50,100 , and 200 starts until at least five starts converged for each GFP trace.

\section{Model selection}

We used the Bayesian information criterion (BIC) (30) for comparing the non-repressor model and the repressor model per total GFP trace. The BIC is calculated by

$$
\mathrm{BIC}=\log (\mathrm{n}) \mathrm{k}-2 \log \mathrm{L}
$$

where $\mathrm{n}$ is the number of data points, $\mathrm{k}$ is the number of estimated parameters and log $\mathrm{L}$ is the log-likelihood value for the maximum likelihood estimate of the model parameters. Here, the number of estimated parameters is either four for the non-repressor model or five for the repressor model. The BIC rewards high likelihood values and penalizes the model complexity in the form of additional model parameters. We considered the repressor model to fit a given total GFP trace considerably better than the non-repressor model if $\mathrm{BIC}_{\text {repressor }}<\mathrm{BIC}_{\text {non-repressor }}$ 10 (Figures 3C-D, 4C-D, S1C-D and S2C-D).

\section{Statistical analysis}

\section{Comparison of initial total GFP of total GFP traces}

On the estimated initial total GFP, GFP, of all total GFP traces significantly better fitted by a repressor model and all total GFP traces better fitted by a non-repressor model, we did a twosided median test. To avoid false-positive results, we used the Bonferroni correction, which adjusts the significance-level $a=0.05$ by the total number of investigated null hypotheses $\mathrm{m}$, such that $a^{\prime}=a / m$. In this study, the total number of null hypotheses for the main analysis is $m=20$ : 
- Two hypothesis tests comparing initial total GFP between repressor and non-repressor fits for wildtype $r 1$ and $r 2$,

- Four hypothesis tests comparing estimated single-cell parameters between wildtype $\mathrm{r} 1$ and r2,

- Two hypothesis tests comparing initial total GFP between repressor and non-repressor fits for elp6 $\Delta \mathrm{r} 1$ and $\mathrm{r} 2$,

- Four hypothesis tests comparing estimated single-cell parameters between elp6 $\Delta \mathrm{r} 1$ and r2,

- Four hypothesis tests comparing estimated single-cell parameters between wildtype and elp6 0 for 1 , and

- Four hypothesis tests comparing estimated single-cell parameters between wildtype and elp6 6 for r2.

In this study, the total number of null hypotheses for the replicate analysis is $m=12$ :

- Two hypothesis tests comparing initial total GFP between repressor and non-repressor fits for wildtype $r 1$ and $r 2$,

- Four hypothesis tests comparing estimated single-cell parameters between wildtype r1 and $\mathrm{r} 2$,

- Two hypothesis tests comparing initial total GFP between repressor and non-repressor fits for elp6 $\Delta \mathrm{r} 1$ and $\mathrm{r} 2$, and

- Four hypothesis tests comparing estimated single-cell parameters between elp6 $\Delta \mathrm{r} 1$ and $\mathrm{r} 2$.

Comparison of estimated single-cell parameters between repression $r 1$ and $r 2$ for wildtype and elp6s

We ignored all traces that were well described by a non-repressor model (uninduced cells) and focused the statistical analysis on the total GFP traces for which the repressor model gave a considerably better fit (see the Model selection, Figures 3C, 4C, S1C and S2C). For those total GFP traces, we compared the estimated single-cell parameters of initial total GFP, GFP, repression delay $t_{\text {delay, }}$, production and degradation rates, $r_{\text {prod }}$ and $r_{\text {deg }}$, for repressions $r 1$ and $r 2$ and wildtype and elp6 $\Delta$. We performed a two-sided paired sign test on the estimated single-cell parameters of paired mother cells in $\mathrm{r} 1$ and $\mathrm{r} 2$ for both wildtype (Figures 3F and S1F) and elp6 (Figures 4F and S2F). To avoid false-positive outcomes, we used the Bonferroni correction, which modified the significance level of $a=0.05$ by the total number of tested null hypotheses $m$ to $a^{\prime}=$ $a / m$, with $m=20$ for the main analysis and $m=12$ for the replicate analysis.

Comparison of estimated single-cell parameters between wildtype and elp6 $\Delta$ for repression $r 1$ and $r 2$

We performed a two-sided median test on the estimated single-cell parameters of all cells for wildtype and elp6 $\Delta$ for $r 1$ (Figure 5B) and r2 (Figure 5D) of the main analysis. Due to the comparison between two different yeast strains, we could not perform a paired test. To counteract false-positive results, we again corrected for multiple testing according to the Bonferroni 
correction, where the significance-level $a=0.05$ was adjusted by the total number of tested null hypotheses $m$ to $a^{\prime}=a / m$ with $m=20$ for the main analysis. As the replicate analysis was performed on pooled data to increase overall cell numbers, we were not able to compare the estimated single-cell parameters between wildtype and elp6 $\Delta$.

\section{Implementation}

The toolboxes used for segmentation, mapping, and tracking are available at https://github.com/gcharvin/phyloCell (PhyloCell (19)), https:/github.com//pbsscientist/YeaZ-GUI (YeaZ (20)), and https://github.com/SchmollerLab/Cell_ACDC (Cell-ACDC (21)). The toolbox used for parameter estimation (PESTO (29)) is available under https://github.com/ICB-DCM. The MATLAB code corresponding to this manuscript is available under https://github.com/marrlab/Gal1repression. The analysis was performed with MATLAB 2017b. 


\section{REFERENCES}

1. M. Acar, A. Becskei, A. van Oudenaarden, Enhancement of cellular memory by reducing stochastic transitions. Nature. 435, 228-232 (2005).

2. K. Liu, Y. Li, V. Prabhu, L. Young, K. G. Becker, P. J. Munson, N.-P. Weng, Augmentation in Expression of Activation-Induced Genes Differentiates Memory from Naive CD4 T Cells and Is a Molecular Mechanism for Enhanced Cellular Response of Memory CD4 T Cells. The Journal of Immunology. 166 (2001), pp. 7335-7344.

3. Y. Araki, Z. Wang, C. Zang, W. H. Wood 3rd, D. Schones, K. Cui, T.-Y. Roh, B. Lhotsky, R. P. Wersto, W. Peng, K. G. Becker, K. Zhao, N.-P. Weng, Genome-wide analysis of histone methylation reveals chromatin state-based regulation of gene transcription and function of memory CD8+ T cells. Immunity. 30, 912-925 (2009).

4. C. V. Mobbs, Glucose-Induced Transcriptional Hysteresis: Role in Obesity, Metabolic Memory, Diabetes, and Aging. Front. Endocrinol. . 9, 232 (2018).

5. L. M. Villeneuve, M. A. Reddy, R. Natarajan, Epigenetics: deciphering its role in diabetes and its chronic complications. Clin. Exp. Pharmacol. Physiol. 38, 451-459 (2011).

6. C. V. Mobbs, Genetic influences on glucose neurotoxicity, aging, and diabetes: a possible role for glucose hysteresis. Genetica. 91, 239-253 (1993).

7. A. J. Courey, S. Jia, Transcriptional repression: the long and the short of it. Genes Dev. 15, 2786-2796 (2001).

8. E. Maldonado, M. Hampsey, D. Reinberg, Repression: targeting the heart of the matter. Cell. 99, 455-458 (1999).

9. H. W. Kosterlitz, The fermentation of galactose and galactose-1-phosphate. Biochemical Journal. 37 (1943), pp. 322-326.

10. L. F. Leloir, The enzymatic transformation of uridine diphosphate glucose into a galactose derivative. Archives of Biochemistry and Biophysics. 33 (1951), pp. 186-190.

11. H. C. Douglas, D. C. Hawthorne, Enzymatic expression and genetic linkage of genes controlling galactose utilization in Saccharomyces. Genetics. 49 (1964), pp. 837-844.

12. D. G. Brickner, I. Cajigas, Y. Fondufe-Mittendorf, S. Ahmed, P.-C. Lee, J. Widom, J. H. Brickner, H2A.Z-Mediated Localization of Genes at the Nuclear Periphery Confers Epigenetic Memory of Previous Transcriptional State. PLoS Biology. 5 (2007), p. e81.

13. S. Kundu, P. J. Horn, C. L. Peterson, SWI/SNF is required for transcriptional memory at the yeast GAL gene cluster. Genes \& Development. 21 (2007), pp. 997-1004.

14. V. Sood, I. Cajigas, A. D'Urso, W. H. Light, J. H. Brickner, Epigenetic Transcriptional Memory of GAL Genes Depends on Growth in Glucose and the Tup1 Transcription Factor in Saccharomyces cerevisiae. Genetics. 206 (2017), pp. 1895-1907.

15. S. R. Stockwell, C. R. Landry, S. A. Rifkin, The yeast galactose network as a quantitative model for cellular memory. Mol. Biosyst. 11, 28-37 (2015).

16. I. Zacharioudakis, T. Gligoris, D. Tzamarias, A yeast catabolic enzyme controls transcriptional memory. Curr. Biol. 17, 2041-2046 (2007). 
17. P. Bheda, D. Aguilar-Gómez, N. B. Becker, J. Becker, E. Stavrou, I. Kukhtevich, T. Höfer, S. Maerkl, G. Charvin, C. Marr, A. Kirmizis, R. Schneider, Single-Cell Tracing Dissects Regulation of Maintenance and Inheritance of Transcriptional Reinduction Memory. Mol. Cell. 78, 915-925.e7 (2020).

18. B. B. Lee, A. Choi, J. H. Kim, Y. Jun, H. Woo, S. D. Ha, C. Y. Yoon, J.-T. Hwang, L. Steinmetz, S. Buratowski, S. Lee, H. Y. Kim, T. Kim, Rpd3L HDAC links H3K4me3 to transcriptional repression memory. Nucleic Acids Res. 46, 8261-8274 (2018).

19. Y. Goulev, S. Morlot, A. Matifas, B. Huang, M. Molin, M. B. Toledano, G. Charvin, Nonlinear feedback drives homeostatic plasticity in $\mathrm{HO}$ stress response. Elife. 6 (2017), doi:10.7554/eLife.23971.

20. N. Dietler, M. Minder, V. Gligorovski, A. M. Economou, D. A. H. L. Joly, A. Sadeghi, C. H. M. Chan, M. Koziński, M. Weigert, A.-F. Bitbol, S. J. Rahi, A convolutional neural network segments yeast microscopy images with high accuracy. Nat. Commun. 11, 5723 (2020).

21. F. Padovani, B. Mairhörmann, P. Falter-Braun, J. Lengefeld, K. M. Schmoller, Cell-ACDC: a user-friendly toolset embedding state-of-the-art neural networks for segmentation, tracking and cell cycle annotations of live-cell imaging data, bioRxiv 2021.09.28.462199, doi:10.1101/2021.09.28.462199.

22. M. Johnston, A model fungal gene regulatory mechanism: the GAL genes of Saccharomyces cerevisiae. Microbiological Reviews. 51 (1987), pp. 458-476.

23. J. Li, S. Wang, W. J. VanDusen, L. D. Schultz, H. A. George, W. K. Herber, H. J. Chae, W. E. Bentley, G. Rao, Green fluorescent protein in Saccharomyces cerevisiae: real-time studies of the GAL1 promoter. Biotechnol. Bioeng. 70, 187-196 (2000).

24. G. I. Lang, D. Botstein, A test of the coordinated expression hypothesis for the origin and maintenance of the GAL cluster in yeast. PLoS One. 6, e25290 (2011).

25. R. K. Niedenthal, L. Riles, M. Johnston, J. H. Hegemann, Green fluorescent protein as a marker for gene expression and subcellular localization in budding yeast. Yeast. 12, 773786 (1996).

26. S. R. Biggar, Cell signaling can direct either binary or graded transcriptional responses. The EMBO Journal. 20 (2001), pp. 3167-3176.

27. I. V. Kukhtevich, N. Lohrberg, F. Padovani, R. Schneider, K. M. Schmoller, Cell size sets the diameter of the budding yeast contractile ring. Nat. Commun. 11, 2952 (2020).

28. H. Hass, C. Loos, E. Raimúndez-Álvarez, J. Timmer, J. Hasenauer, C. Kreutz, Benchmark problems for dynamic modeling of intracellular processes. Bioinformatics. 35 (2019), pp. 3073-3082.

29. P. Stapor, D. Weindl, B. Ballnus, S. Hug, C. Loos, A. Fiedler, S. Krause, S. Hroß, F. Fröhlich, J. Hasenauer, PESTO: Parameter EStimation TOolbox. Bioinformatics. 34, 705-707 (2018).

30. G. Schwarz, Estimating the Dimension of a Model. The Annals of Statistics. 6 (1978), , doi:10.1214/aos/1176344136. 
A

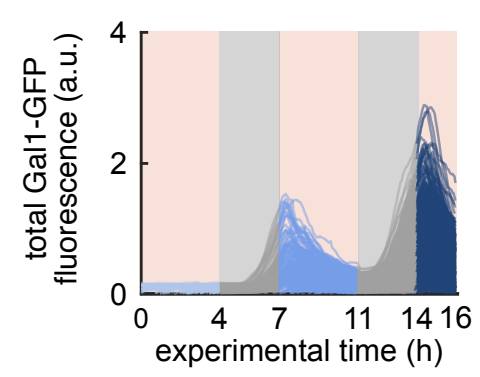

B

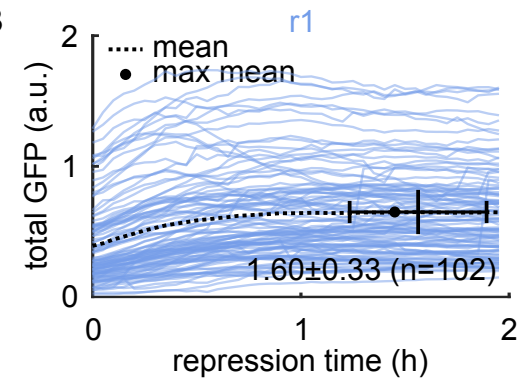

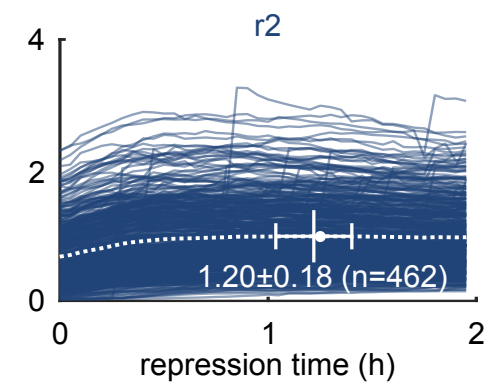

r2

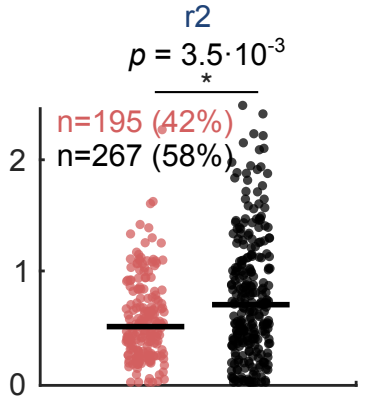

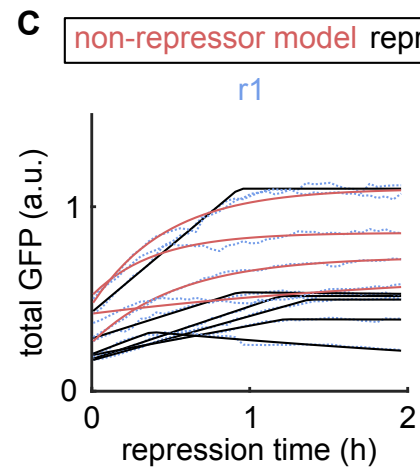

D
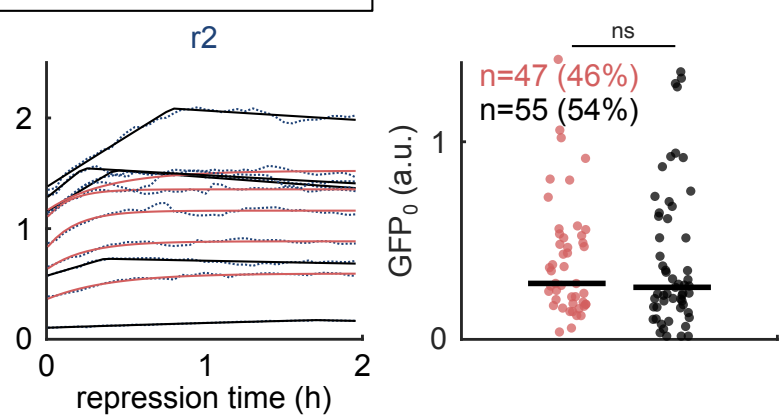

$\mathbf{F}$

E $n=55$

$\mathrm{n}=267$

F $\quad p=\underset{* \star \star}{4.6 \cdot 10^{-7}} \quad p=\underset{\star}{3.3 \cdot 10^{-3}}$
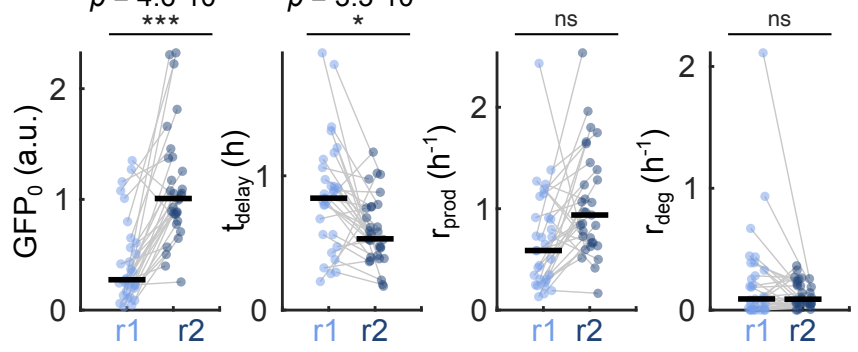

Figures 2 and 3 - Figure S1. Earlier repression response in repression $r 2$ at both the population and single-cell level for cells of replicate experiment.

(A) Single-cell traces of total Gal1-GFP fluorescence signal across two inductions i1 and i2 (gray) and repressions $\mathrm{r} 0, \mathrm{r} 1$, and $\mathrm{r} 2$ (blue).

(B) Single-cell traces of total GFP signal adjusted for dilution (see (C)) for the first two hours of repression $\mathrm{r} 1$ (left) and repression $\mathrm{r} 2$ (right). Time to maximal mean total GFP is 24 min shorter in repression r2, where mean total GFP is indicated by the dotted line and the maximal mean total GFP is highlighted by the dot. Bootstrap $\left(10^{5}\right)$ samples were drawn to generate mean \pm std.

(C) Ten exemplary total GFP traces (dotted lines) and best fits (solid lines) for repressions $r 1$ (left) and r2 (right). GFP traces best fitted with a repressor model are shown in black and fits of total GFP traces best fitted with a non-repressor model are shown in red.

(D) The median initial total GFP, GFP, is higher in traces better fitted by the repressor model (black) than in traces better fitted by the non-repressor model (red). This confirms that the repressor model fits induced cells better, while the non-repressor model fits uninduced cells. The number of cells and percentages of all GFP traces best fitted by the repressor model and non-repressor model are shown.

(E) Time to maximal mean total GFP is decreased in repression r2 for repressor cells $(0.97 \pm 0.08$ vs. 0.88 $\pm 0.04)$. Bootstrap $\left(10^{5}\right)$ samples of the repressor cells were drawn to generate mean \pm std.

(F) Comparison of paired estimated single-cell parameters of repression $r 1$ and $r 2$ shows that the median initial total GFP, GFP, and median repression delay, tdelay, are significantly different $\left(p=4.6 \cdot 10^{-7}\right.$ and $p=$ $3.3 \cdot 10^{-3}$, respectively, two-sided paired sign test correcting for multiple testing with Bonferroni correction, $\mathrm{m}=12$, and the number of paired cells $=31$ ), with median GFPo increased and median tdelay decreased (median $t_{\text {delay }}$ values of 0.83 and 0.53 for $\mathrm{r} 1$ and $\mathrm{r} 2$, respectively). Median production rate, $\mathrm{r}_{\text {prod, }}$ and median degradation rate, $r_{d e g}$, are not significantly different between $r 1$ and $r 2(p=0.01$ and $p=0.50$, respectively). 
A

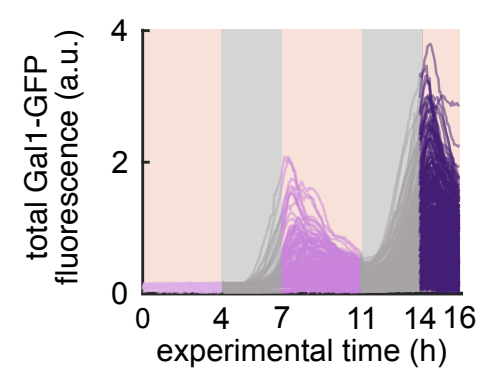

B

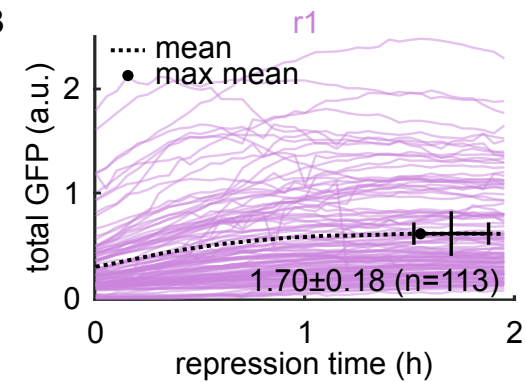

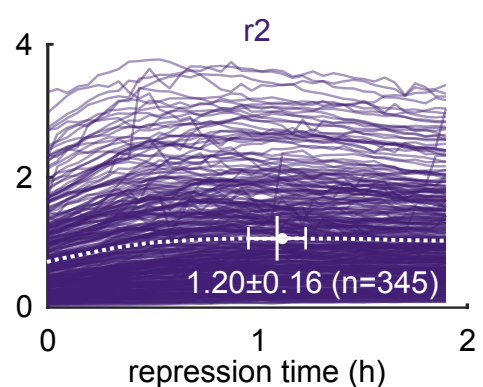

r2

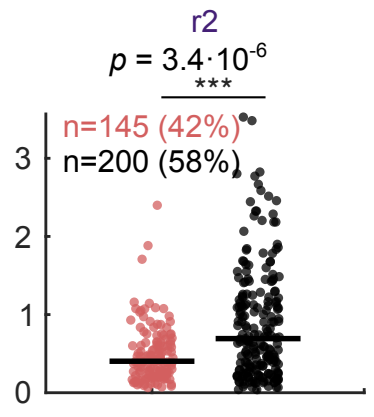

C

non-repressor model repressor model ...... data — fit
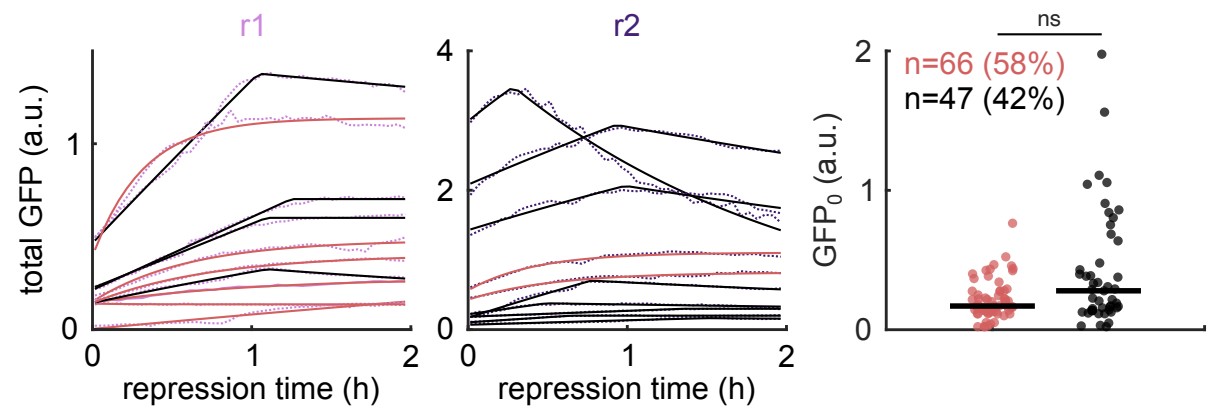

$\mathbf{F}$

$n=47$
$n=200$
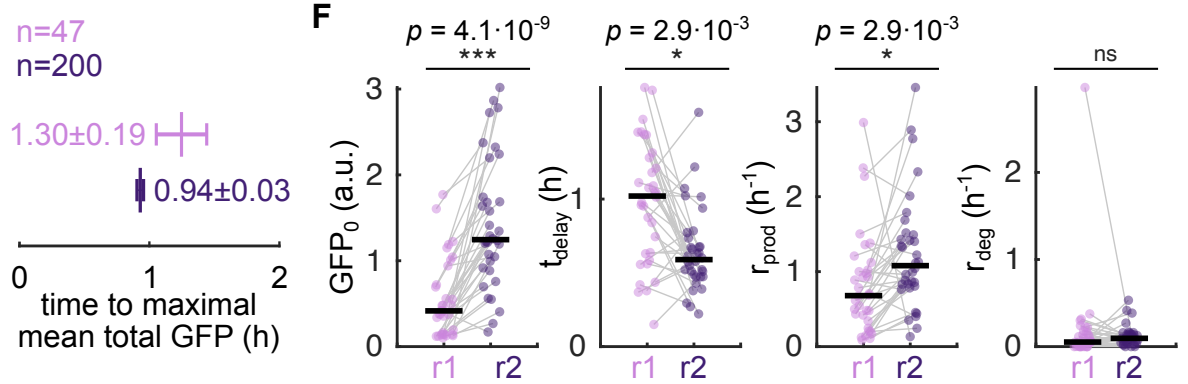

Figure 4 - Figure S2. Earlier repression response in repression $r 2$ at both the population and singlecell level for elp $6 \Delta$ cells of replicate experiment.

(A) Single-cell traces of total Gal1-GFP fluorescence signal of elp6 budding yeast cells across two inductions i1 and i2 (gray) and repressions 0, 1, and 2 (purple).

(B) Single-cell traces of total GFP signal of elp6 $\Delta$ budding yeast cells adjusted for dilution (see Figure $2 \mathrm{C}$ ) for the first two hours of $\mathrm{r} 1$ (left) and $\mathrm{r} 2$ (right). Time to maximal mean total GFP is 36 min shorter in repression $\mathrm{r}$, where the mean total GFP signal is indicated by the dotted line and the maximal mean total GFP is highlighted by the dot. Bootstrap $\left(10^{5}\right)$ samples were drawn to generate mean \pm std.

(C) Ten exemplary total GFP traces (dotted lines) and best fits (solid lines) of elp6 $\Delta$ budding yeast cells and repressions $\mathrm{r} 1$ (left) and $\mathrm{r} 2$ (right).

(D) The median initial total GFP, GFPo, is higher in elp6 $\Delta$ traces better fitted by the repressor model (black) than in elp6 $\Delta$ traces better fitted by the non-repressor model (red). This confirms that the repressor model fits induced elp6 $\Delta$ cells better, while the non-repressor model fits uninduced elp6 $\Delta$ cells. The number of cells and percentages of all GFP traces best fitted by the repressor model and non-repressor model are shown.

(E) Time to maximal mean total GFP is decreased in repression r2 for elp6 $\Delta$ repressor cells $(1.30 \pm 0.19$ vs. $0.94 \pm 0.02)$. Bootstrap $\left(10^{5}\right)$ samples of the repressor cells were drawn to generate mean \pm std.

(F) Comparison of paired estimated single-cell parameters of elp6 $\Delta$ cells of repression $r 1$ and $r 2$ show that median initial total GFP, GFP, median repression delay, tdelay, and median production rate, $\mathrm{r}_{\text {prod, }}$ are significantly different $\left(p=4.1 \cdot 10^{-9}, p=2.9 \cdot 10^{-3}\right.$, and $p=2.9 \cdot 10^{-3}$, respectively, two-sided paired sign test correcting for multiple testing with Bonferroni correction, $m=12$, and the number of paired cells is 34), with median GFP 0 and $r_{\text {prod }}$ increased and median tdelay decreased (median values of 1.00 and $0.59 \mathrm{~h}$ for $\mathrm{r} 1$ and r2, respectively) in r2. Median degradation rate, $r_{d e g}$, is not significantly different between $r 1$ and $r 2(p=$ $0.39)$. 